PROCEEDINGS OF THE

AMERICAN MATHEMATICAL SOCIETY

Volume 138, Number 7, July 2010, Pages 2397-2402

S 0002-9939(10)10352-9

Article electronically published on February 18, 2010

\title{
ANOTHER PROOF FOR THE REMOVABLE SINGULARITIES OF THE HEAT EQUATION
}

\author{
KIN MING HUI
}

(Communicated by Yingfei Yi)

\begin{abstract}
We give two different simple proofs for the removable singularities of the heat equation in $\left(\Omega \backslash\left\{x_{0}\right\}\right) \times(0, T)$, where $x_{0} \in \Omega \subset \mathbb{R}^{n}$ is a bounded domain with $n \geq 3$. We also give a necessary and sufficient condition for removable singularities of the heat equation in $\left(\Omega \backslash\left\{x_{0}\right\}\right) \times(0, T)$ for the case $n=2$.
\end{abstract}

Singularities of solutions of partial differential equations appear in many problems. For example, singularities appear in the study of the solutions of the harmonic map $[13$ and the harmonic map heat flow [3]. In 14] S. Sato and E. Yanagida studied the solutions for a semilinear parabolic equation with moving singularities. Singularities of solutions also appear in the study of hyperbolic partial differential equations $[15$ and in the study of the touchdown behavior of the micro-electromechanical systems equation [4, 6], [5].

It is interesting to find a necessary and sufficient condition for the solutions of the equations to have removable singularities. In 8 S.Y. Hsu proved the following theorem.

Theorem 1. Let $n \geq 3$ and let $0 \in \Omega \subset \mathbb{R}^{n}$ be a domain. Suppose $u$ is a solution of the heat equation

$$
u_{t}=\Delta u
$$

in $(\Omega \backslash\{0\}) \times(0, T)$. Then $u$ has removable singularities at $\{0\} \times(0, T)$ if and only if for any $0<t_{1}<t_{2}<T$ and $\delta \in(0,1)$ there exists $\overline{B_{R_{0}}(0)} \subset \Omega$ depending on $t_{1}$, $t_{2}$ and $\delta$, such that

$$
|u(x, t)| \leq \delta|x|^{2-n}
$$

for any $0<|x| \leq R_{0}$ and $t_{1} \leq t \leq t_{2}$.

The proof in [8] is based on the Green function estimates of [9] and a careful analysis of the behavior of the solution near the singularities using the Duhamel principle. In this paper we will use the Schauder estimates for the heat equation [2], [12, and the technique of [1] and [7] to give two different simple proofs of the above result. We also obtain the following result for the solution of the heat equation in two dimensions.

Received by the editors September 1, 2009, and, in revised form, September 2, 2009.

2010 Mathematics Subject Classification. Primary 35B65; Secondary 35K05.

Key words and phrases. Removable singularities, heat equation.

(C)2010 American Mathematical Society Reverts to public domain 28 years from publication 
Theorem 2. Let $0 \in \Omega \subset \mathbb{R}^{2}$ be a domain. Suppose $u$ is a solution of the heat equation in $(\Omega \backslash\{0\}) \times(0, T)$. Then $u$ has removable singularities at $\{0\} \times(0, T)$ if and only if for any $0<t_{1}<t_{2}<T$ and $\delta \in(0,1)$ there exists $\overline{B_{R_{0}}(0)} \subset \Omega$ depending on $t_{1}, t_{2}$ and $\delta$ such that

$$
|u(x, t)| \leq \delta(\log (1 /|x|))^{-1}
$$

for any $0<|x| \leq R_{0}$ and $t_{1} \leq t \leq t_{2}$.

Remark 3. Note that the function $\log |x|$ satisfies the heat equation in $\left(\mathbb{R}^{2} \backslash\{0\}\right) \times$ $(0, \infty)$, but it has non-removable singularities on $\{0\} \times(0, \infty)$ and it does not satisfy (3). Hence (3) is sharp.

We start with some definitions. For any set $A$ we let $\chi_{A}$ be the characteristic function of the set $A$. Let $0 \in \Omega \subset \mathbb{R}^{n}$ be a bounded domain. We say that a solution $u$ of the heat equation $(1)$ in $(\Omega \backslash\{0\}) \times(0, T)$ has removable singularities at $\{0\} \times(0, T)$ if there exists a classical solution $v$ of $(1)$ in $\Omega \times(0, T)$ such that $u=v$ in $(\Omega \backslash\{0\}) \times(0, T)$. For any $R>0$ let $B_{R}=B_{R}(0)=\{x:|x|<R\} \subset \mathbb{R}^{n}$.

Proof of Theorem 1. Suppose $u$ has removable singularities in $\{0\} \times(0, T)$. By the same argument as in the proof in section 3 of [8] for any $0<t_{1}<t_{2}<T$ and $\delta \in(0,1)$ there exists $\bar{B}_{R_{0}} \subset \Omega$ depending on $t_{1}, t_{2}$ and $\delta$ such that (2) holds.

Suppose (2) holds. Then for any $0<t_{1}<t_{2}<T$ and $\delta \in(0,1)$ there exists $\bar{B}_{R_{0}} \subset \Omega$ depending on $t_{1}, t_{2}$ and $\delta$ such that (2) holds for any $0<|x| \leq R_{0}$ and $t_{1} \leq t \leq t_{2}$.

For any $0<|x| \leq R_{0}$, let

$$
w(y, s)=u\left(|x| y,|x|^{2} s\right) \quad \forall 0<|y| \leq R_{0} /|x|, t_{1} /|x|^{2} \leq s \leq t_{2} /|x|^{2} .
$$

Then $w$ is a solution of (1) in $\left(\bar{B}_{1} \backslash\{0\}\right) \times\left(|x|^{-2} t_{1},|x|^{-2} t_{2}\right)$. By (2),

$$
|w(y, s)| \leq \delta(|x||y|)^{2-n} \quad \forall 0<|y| \leq R_{0} /|x|, t_{1} /|x|^{2} \leq s \leq t_{2} /|x|^{2} .
$$

Let $t_{1}<t_{3}<t_{2}$. Then

$$
\frac{t_{3}}{|x|^{2}}-\frac{t_{1}}{|x|^{2}} \geq \frac{t_{3}-t_{1}}{R_{0}^{2}} .
$$

By the parabolic Schauder estimates 2], 12, (5) and (6), there exists a constant $C_{1}>0$ such that

$$
|\nabla w(y, s)| \leq C_{1} \quad \sup _{\substack{1 / 2 \leq \leq\left. z|\leq 1\\| x\right|^{-2} t_{1} \leq \tau \leq|x|^{-2} t_{2}}} w(z, \tau) \leq C_{2} \delta|x|^{2-n}
$$

holds for any $2 / 3 \leq|y| \leq 3 / 4, t_{3} /|x|^{2} \leq s \leq t_{2} /|x|^{2}$, where $C_{2}=2^{n-2} C_{1}$. By (4) and (7),

$$
\begin{aligned}
|\nabla u(z, t)| \leq C_{2} \delta|x|^{1-n} & \forall|z| & =\frac{3}{4}|x|, 0<|x| \leq R_{0}, t_{3} \leq t \leq t_{2} \\
\Rightarrow & |\nabla u(z, t)| \leq C_{2} \delta|z|^{1-n} \quad \forall|z| & \leq \frac{3}{4} R_{0}, t_{3} \leq t \leq t_{2} .
\end{aligned}
$$

Let $R_{1}=3 /\left(4 R_{0}\right)$. We will now use a modification of the proof of Lemma 2.3 of [1] and Lemma 2.1 of [7] to complete the argument. We will first show that $u$ satisfies 
(1) in $\Omega \times\left(t_{1}, t_{2}\right)$ in the distribution sense. Since $u$ satisfies $(1)$ in $(\Omega \backslash\{0\}) \times(0, T)$, for any $0<\varepsilon<R_{1}$ and $\eta \in C_{0}^{\infty}(\Omega \times(0, T))$ we have

$$
\begin{aligned}
\left.\int_{\Omega \backslash B_{\varepsilon}} u \eta d x\right|_{t_{3}} ^{t_{2}}=\int_{t_{3}}^{t_{2}} & \int_{\Omega \backslash B_{\varepsilon}} u \eta_{t} d x d t-\int_{t_{3}}^{t_{2}} \int_{\Omega \backslash B_{\varepsilon}} \nabla u \cdot \nabla \eta d x d t \\
& -\int_{t_{3}}^{t_{2}} \int_{\partial B_{\varepsilon}} \eta \frac{\partial u}{\partial n} d \sigma d t
\end{aligned}
$$

where $\partial u / \partial n$ is the derivative of $u$ with respect to the unit outward normal at $\partial B_{\varepsilon}$. By (8),

$$
\limsup _{\varepsilon \rightarrow 0}\left|\int_{t_{3}}^{t_{2}} \int_{\partial B_{\varepsilon}} \eta \frac{\partial u}{\partial n} d \sigma d t\right| \leq C_{2} \delta\left(t_{2}-t_{3}\right)\left|\partial B_{1}\right|\|\eta\|_{L^{\infty}} .
$$

Since $\delta>0$ is arbitrary, it follows that

$$
\lim _{\varepsilon \rightarrow 0} \int_{t_{3}}^{t_{2}} \int_{\partial B_{\varepsilon}} \eta \frac{\partial u}{\partial n} d \sigma d t d x d t=0 .
$$

By (8) and the Lebesgue dominated convergence theorem,

$$
\lim _{\varepsilon \rightarrow 0} \int_{t_{3}}^{t_{2}} \int_{\Omega \backslash B_{\varepsilon}} \nabla u \cdot \nabla \eta d x d t=\int_{t_{3}}^{t_{2}} \int_{\Omega} \nabla u \cdot \nabla \eta d x d t .
$$

Letting $\varepsilon \rightarrow 0$ in (9), by (10) and (11) it follows that

$$
\left.\int_{\Omega} u \eta d x\right|_{t_{3}} ^{t_{2}}=\int_{t_{3}}^{t_{2}} \int_{\Omega} u \eta_{t} d x d t-\int_{t_{3}}^{t_{2}} \int_{\Omega} \nabla u \cdot \nabla \eta d x d t \quad \forall t_{3} \in\left(t_{1}, t_{2}\right) .
$$

Hence $u$ is a distribution solution of (1) in $\Omega \times\left(t_{1}, t_{2}\right)$. By (2), for any $1 \leq p<\frac{n}{n-2}$ there exists a constant $C_{p}^{\prime}>0$ such that

$$
\sup _{t_{1} \leq t \leq t_{2}} \int_{B_{R_{0}}} u(x, t)^{p} d x \leq C_{p}^{\prime} .
$$

By (12) and (13) and an argument similar to the proof of [11] and section 1 of [10, $u \in L_{l o c}^{\infty}\left(B_{R_{0}} \times\left(t_{1}, t_{2}\right)\right)$. We now let $v$ be the solution of

$$
\begin{cases}v_{t}=\Delta v & \text { in } B_{R_{1}} \times\left(t_{3}, t_{2}\right), \\ \frac{\partial v}{\partial n}(x, t)=\frac{\partial u}{\partial n}(x, t) & \text { on } \partial B_{R_{1}} \times\left(t_{3}, t_{2}\right), \\ v\left(x, t_{3}\right)=u\left(x, t_{3}\right) & \text { in } B_{R_{1}} .\end{cases}
$$

For any $0 \leq h \in C_{0}^{\infty}\left(B_{R_{1}}\right)$ and $t_{3}<t \leq t_{2}$ let $\eta$ be the solution of

$$
\left\{\begin{array}{l}
\eta_{t}+\Delta \eta=0 \quad \text { in } B_{R_{1}} \times\left(t_{3}, t\right), \\
\frac{\partial \eta}{\partial n}(x, t)=0 \quad \text { on } \partial B_{R_{1}} \times\left(t_{3}, t\right), \\
\eta(x, t)=h(x) \quad \text { in } B_{R_{1}} .
\end{array}\right.
$$

By the maximum principle,

$$
0 \leq \eta \leq\|h\|_{L^{\infty}} \quad \text { in } B_{R_{1}} \times\left(t_{3}, t\right) .
$$


Then by (14) and (15),

$$
\begin{aligned}
\left.\int_{B_{R_{1}} \backslash B_{\varepsilon}}(u-v) \eta d x\right|_{t_{3}} ^{t}= & \int_{t_{3}}^{t} \int_{B_{R_{1} \backslash B_{\varepsilon}}}\left[(u-v) \eta_{t}+(u-v)_{t} \eta\right] d x d t \\
= & \int_{t_{3}}^{t} \int_{B_{R_{1} \backslash B_{\varepsilon}}}\left[(u-v) \eta_{t}+\Delta(u-v) \eta\right] d x d t \\
= & \int_{t_{3}}^{t} \int_{B_{R_{1} \backslash B_{\varepsilon}}(u-v)\left(\eta_{t}+\Delta \eta\right) d x d t} \\
& -\int_{t_{3}}^{t} \int_{\partial B_{\varepsilon}} \eta \frac{\partial}{\partial n}(u-v) d \sigma d t+\int_{t_{3}}^{t} \int_{\partial B_{\varepsilon}}(u-v) \frac{\partial \eta}{\partial n} d \sigma d t \\
= & -\int_{t_{3}}^{t} \int_{\partial B_{\varepsilon}} \eta \frac{\partial}{\partial n}(u-v) d \sigma d t+\int_{t_{3}}^{t} \int_{\partial B_{\varepsilon}}(u-v) \frac{\partial \eta}{\partial n} d \sigma d t
\end{aligned}
$$

By (2),

$$
\left|\int_{t_{3}}^{t} \int_{\partial B_{\varepsilon}}(u-v) \frac{\partial \eta}{\partial n} d \sigma d t\right| \leq C \varepsilon \rightarrow 0 \quad \text { as } \varepsilon \rightarrow 0 .
$$

By (8) and (16),

$$
\limsup _{\varepsilon \rightarrow 0}\left|\int_{t_{3}}^{t} \int_{\partial B_{\varepsilon}} \eta \frac{\partial}{\partial n}(u-v) d \sigma d t\right| \leq C \delta .
$$

Since $\delta>0$ is arbitrary, by (19) it follows that

$$
\lim _{\varepsilon \rightarrow 0}\left|\int_{t_{3}}^{t} \int_{\partial B_{\varepsilon}} \eta \frac{\partial}{\partial n}(u-v) d \sigma d t\right|=0
$$

Letting $\varepsilon \rightarrow 0$ in (17), by (18) and (20),

$$
\int_{B_{R_{1}}}(u-v)(x, t) h(x) d x=\int_{B_{R_{1}}}(u-v)\left(x, t_{3}\right) \eta\left(x, t_{3}\right) d x=0 .
$$

We now choose a sequence of functions $h_{i} \in C_{0}^{\infty}\left(B_{R_{1}}\right)$ converging to $\chi_{\{u>v\}}$ a.e. $x \in B_{R_{1}}$ as $i \rightarrow \infty$. Putting $h=h_{i}$ in (21) and letting $i \rightarrow 0$,

$$
\int_{B_{R_{1}}}(u-v)_{+}(x, t) d x=0 \quad \forall t_{3}<t \leq t_{2} .
$$

By interchanging the roles of $u$ and $v$ we get

$$
\int_{B_{R_{1}}}(v-u)_{+}(x, t) d x=0 \quad \forall t_{3}<t \leq t_{2} .
$$

Hence by (22) and (23),

$$
\begin{gathered}
\int_{B_{R_{1}}}|v-u|(x, t) d x=0 \quad \forall t_{3}<t \leq t_{2} \\
\Rightarrow \quad u(x, t)=v(x, t) \quad \forall 0<|x| \leq R_{1}, t_{3}<t \leq t_{2} .
\end{gathered}
$$

Hence $u$ has removable singularities on $\{0\} \times\left(t_{3}, t_{2}\right)$. Since $0<t_{1}<t_{3}<t_{2}<T$ is arbitrary, $u$ has removable singularities on $\{0\} \times(0, T)$ and the theorem follows.

Proof of Theorem 2. Theorem 2 follows by an argument very similar to the proof of Theorem 1 but with (3) replacing (2) in the argument. 
An alternate proof of Theorems 1 and 2. We will show that when (2) (respectively (3)) holds, then $u$ has removable singularities at $\{0\} \times(0, T)$. Suppose $(2)$ holds if $n \geq 3$ and (3) holds if $n=2$. We first observe that by the previous argument, for any $0<t_{1}<t_{2}<T$, $u$ satisfies (12) and $u \in L_{l o c}^{\infty}(\Omega \times(0, T))$. Let $\bar{B}_{R_{1}} \subset \Omega$ and let $w$ be the solution of

$$
\begin{cases}w_{t}=\Delta w & \text { in } B_{R_{1}} \times\left(t_{1}, t_{2}\right), \\ w=u & \text { on } \bar{B}_{R_{1}} \times\left\{t_{1}\right\} \cup \partial B_{R_{1}} \times\left(t_{1}, t_{2}\right) .\end{cases}
$$

By the maximum principle,

$$
\|w\|_{L^{\infty}} \leq\|u\|_{L^{\infty}\left(B_{R_{1}} \times\left(t_{1}, t_{2}\right)\right)}<\infty .
$$

For any $\varepsilon>0$, let

$$
w_{\varepsilon}= \begin{cases}w-u+\varepsilon|x|^{2-n} & \text { if } n \geq 3, \\ w-u+\varepsilon \log \left(R_{1} /|x|\right) & \text { if } n=2 .\end{cases}
$$

Then $w_{\varepsilon}$ satisfies

$$
\begin{cases}w_{\varepsilon, t}=\Delta w_{\varepsilon} & \text { in }\left(B_{R_{1}} \backslash\{0\}\right) \times\left(t_{1}, t_{2}\right), \\ w_{\varepsilon} \geq u & \text { on } \partial B_{R_{1}} \times\left(t_{1}, t_{2}\right) \cup \bar{B}_{R_{1}} \times\left\{t_{1}\right\} .\end{cases}
$$

By (2), (3), and (25) there exists a constant $0<r_{0}<R_{1}$ such that

$$
w_{\varepsilon} \geq 0 \quad \text { on } \partial B_{r_{1}} \times\left[t_{1}, t_{2}\right]
$$

for all $0<r_{1} \leq r_{0}$. By the maximum principle in $\left(B_{R_{1}} \backslash B_{r_{1}}\right) \times\left(t_{1}, t_{2}\right)$,

$$
\begin{aligned}
& w_{\varepsilon} \geq 0 \quad \text { in }\left(B_{R_{1}} \backslash B_{r_{1}}\right) \times\left(t_{1}, t_{2}\right) \\
\Rightarrow & \begin{cases}w-u+\varepsilon|x|^{2-n} \geq 0 \quad \forall r_{1} \leq|x| \leq R_{1}, t_{1} \leq t \leq t_{2} \quad \text { if } n \geq 3, \\
w-u+\varepsilon \log \left(R_{0} /|x|\right) \geq 0 \quad \forall r_{1} \leq|x| \leq R_{1}, t_{1} \leq t \leq t_{2} \quad \text { if } n=2\end{cases} \\
\Rightarrow & w \geq u \quad \forall 0<|x| \leq R_{1}, t_{1} \leq t \leq t_{2} \quad \text { as } r_{1} \rightarrow 0, \varepsilon \rightarrow 0 .
\end{aligned}
$$

Similarly, by considering the function

$$
v_{\varepsilon}=\left\{\begin{array}{lc}
w-u-\varepsilon|x|^{2-n} & \text { if } n \geq 3, \\
w-u-\varepsilon \log \left(R_{1} /|x|\right) & \text { if } n=2
\end{array}\right.
$$

and applying the maximum principle and letting $\varepsilon \rightarrow 0$, we get

$$
w \leq u \quad \forall 0<|x| \leq R_{1}, t_{1} \leq t \leq t_{2} .
$$

By (26) and (27) we get (24), and Theorem 1 and Theorem 2 follow.

\section{REFERENCES}

[1] B.E.J. Dahlberg and C. Kenig, Nonnegative solutions of generalized porous medium equations, Revista Matemática Iberoamericana 2 (1986), 267-305. MR.908054 (88k:35223)

[2] A. Friedman, Partial differential equations of parabolic type, Prentice-Hall, Inc., Englewood Cliffs, N.J., 1964. MR0181836 (31:6062)

[3] M. Guan, S. Gustafson and T.-P. Tsai, Global existence and blow-up for harmonic map heat flow, J. Differential Equations 246 (2009), no. 1, 1-20. MR2467012(2009i:35154)

[4] N. Ghoussoub and Y.J. Guo, On the partial differential equations of electrostatic MEMS devices. II: Dynamic case, NoDEA Nonlinear Differential Equations Appl. 15 (2008), no. 1-2, 115-145. MR2408347(2009i:35153)

[5] N. Ghoussoub and Y.J. Guo, Estimates for the quenching time of a parabolic equation modeling electrostatic MEMS, Methods Appl. Anal. 15 (2008), 361-376. MR2500853 
[6] Y.J. Guo, On the partial differential equations of electrostatic MEMS devices. III: Refined touchdown behavior, J. Diff. Eqns. 244 (2008), no. 9, 2277-2309. MR2413842 (2009i:35155)

[7] S.Y. Hsu, Asymptotic behaviour of solutions of the equation $u_{t}=\Delta \log u$ near the extinction time, Advances in Differential Equations 8 (2003), no. 2, 161-187. MR1948043 (2003i:35133)

[8] S.Y. Hsu, Removable singularities of semilinear parabolic equations, Advances in Differential Equations 15 (2010), no. 1-2, 137-158.

[9] K.M. Hui, A Fatou theorem for the solution of the heat equation at the corner points of a cylinder, Trans. Amer. Math. Soc. 333 (1992), no. 2, 607-642. MR1091707 (92m:35106)

[10] K.M. Hui, Existence of solutions of the equation $u_{t}=\Delta \log u$, Nonlinear Analysis TMA 37 (1999), no. 7, 875-914. MR1695083 (2000c:35131)

[11] S. Kuang and Q.S. Zhang, A gradient estimate for all positive solutions of the conjugate heat equation under Ricci flow, J. Funct. Anal. 255 (2008), no. 4, 1008-1023. MR2433960 (2009h:53150)

[12] O.A. Ladyzenskaya, V.A. Solonnikov, and N.N. Uraltceva, Linear and quasilinear equations of parabolic type, Transl. Math. Mono., Vol. 23, Amer. Math. Soc., Providence, R.I., 1968.

[13] J. Sacks and K. Uhlenbeck, The existence of minimal immersions of 2-spheres, Ann. of Math. (2) 113 (1981), no. 1, 1-24. MR604040 (82f:58035)

[14] S. Sato and E. Yanagida, Solutions with moving singularities for a semilinear parabolic equation, J. Differential Equations 246 (2009), no. 2, 724-748. MR2468735 (2009m:35242)

[15] J. Smoller, Shock waves and reaction-diffusion equations, Springer-Verlag, New York, 1994. MR1301779 (95g:35002)

Institute of Mathematics, Academia Sinica, Nankang, Taipei, 11529, Taiwan, Republic OF CHINA 